\title{
Etude de l'impact des barrages sur la réduction des transports sédimentaires jusqu'à la mer par approche paléohydrologique dans la basse vallée de la Medjerda
}

\author{
Fatma Kotti $^{1}$, Laurent Dezileau ${ }^{3}$, Gil Mahé ${ }^{2}$, Hamadi Habaieb $^{1}$, Malik Bentkaya ${ }^{4}$, Claudine Dieulin $^{2}$, \\ and Oula Amrouni ${ }^{5}$ \\ ${ }^{1}$ Institut National Agronomique de Tunis, 43, Av. Charles Nicolle 1082 Tunis-Mahrajène, Tunisie \\ ${ }^{2}$ UMR HydroSciences Montpellier/IRD, Montpellier, France \\ ${ }^{3}$ Géoscience Montpellier, Université de Montpellier, Montpellier, France \\ ${ }^{4}$ Centre National des Sciences et Technologies Nucléaires, Sidi Thabet, Tunisie \\ ${ }^{5}$ Institut National des Sciences et Technologies de la Mer, Salammbô, Tunisie
}

Correspondence: Fatma Kotti (f.kotti@yahoo.fr)

Received: 15 June 2017 - Accepted: 4 October 2017 - Published: 16 April 2018

Résumé. The sedimentary contributions of the Medjerda to the coastal zone are poorly measured, and there is no chronicle of observations. In this context, the sediment monitoring appears indispensable for the quantification of sediment transport at the outlet. This study focuses on the largest watershed in Tunisia, the Wadi Medjerda $\left(23600 \mathrm{~km}^{2}\right)$. The main objective of this work is to assess the reduction of sediment transport following anthropogenic intensification on the basin, especially since the construction of many large dams. In order to collect information on actual deposits over several years, the paleo-hydrological approach was applied through the study of sediment cores sampled in the low valley meanders on alluvial terraces, after the last dam (Sidi Salem, the largest water storage capacity over the basin), but before the estuary to avoid marine influence and near a hydrological station (Jdaida). The sedimentary deposits of the river provide key information on the past sedimentary inputs. A visible succession of sedimentary layers corresponding to the deposits of successive floods on the study site has been determined and the history of the sedimentary contributions of the Medjerda is reconstructed by this approach. The thickest layers of sedimentary deposits are related to exceptional events. They are mainly concentrated on the lower part of the core and are mainly composed of sands. The first $1.2 \mathrm{~m}$ of the core from the bottom upward relates to 10 years of river discharges, as can be determined from the ${ }^{137} \mathrm{Cs}$ datation. The next upward $1.05 \mathrm{~m}$ of core relates to the following 20 years of discharges, up to 1981, date of the construction of the Sidi Salem dam, and is composed of a mix of sand, silts and clays. The last $75 \mathrm{~cm}$ of core near the surface is only composed of clays with thin silt bands, and relates to a period of 32 years. We thus observe that there is no more sand deposits in the river bed since the construction of the Sidi Salem dam. The deficit of sediment supply to the sea is viewed as a major factor to be taken into account for better understanding of the dynamics of coastal areas in the context of global climate change. 


\section{Introduction}

Dans le contexte actuel des changements climatiques, le suivi du transport sédimentaire dans les rivières est important pour mettre l'accent sur les problèmes environnementaux souvent reliés à des changements observés dans le cycle hydrologique : modification de la distribution des précipitations, du ruissellement et de l'intensité des extrêmes hydrologiques (Bates et al., 2008 ; Hungtinton, 2006 ; Labat et al., 2004 ; Solomon, 2007). Le suivi du transport sédimentaire permet également d'évaluer et de quantifier les transferts de matières des continents vers les océans. Cependant, le problème du nombre réduit de données relatives aux transports solides a toujours freiné les investigations. Les mesures de transport solide effectuées en Tunisie et les études ponctuelles détaillées restent insuffisantes pour traiter ce sujet (Ghorbel et Claude, 1982). Le défi consiste donc à utiliser des méthodes de mesures simples pour combler le manque de connaissances. La présente étude s'intéresse au fleuve Medjerda en Tunisie, afin d'étudier en détail l'évolution des transports solides à l'exutoire de son bassin versant. L'Oued Medjerda est devenu un fleuve presque totalement contrôlé depuis l'intensification des aménagements sur le bassin au début du XXème siècle, pour atténuer les effets des crues dans la basse vallée très intensément exploitée. Le transport de matières solides a été considérablement réduit dans sa basse vallée, en particulier depuis l'ouverture du barrage Sidi Salem sur le cours principal de la Medjerda, en 1981, responsable d'une modification majeure du régime hydrologique du fleuve en aval (Zahar et al., 2008). L'alluvionnement des retenues des aménagements constitue un problème récent et majeur (Ben Mamou et Louati, 2007). Or, l'histoire des apports sédimentaires dans cette zone d'étude n'est pas connue, ce qui entraîne deux difficultés :

- comment évaluer l'apport solide à la mer si l'histoire des apports sédimentaires n'est pas connue ?

- comment identifier les différents facteurs contrôlant l'apport sédimentaire par la Medjerda ?

La paléohydrologie peut en partie répondre à ces questions. On tentera dans ce cas d'établir un historique de la sédimentation dans le bief aval du fleuve Medjerda, avant la mer, et de relier cet historique avec l'histoire des débits, l'évolution climatique et la construction des aménagements. La paléohydrologie est la reconstruction de l'ampleur et de la fréquence des inondations récentes et passées en utilisant des archives sédimentaires (Baker et al., 2002). Notre travail va se concentrer principalement sur la Medjerda, près de la station hydrologique de Jdaida située dans la basse vallée mais suffisamment loin de la mer pour ne pas être impactée par la marée, zone d'étude pour laquelle il existe une quantité importante de données historiques, particulièrement sur les débits des 70 dernières années. Notre approche consiste à prélever des carottes de sédiment dans un méandre où les dépôts sont ré- guliers et peu turbulents, afin de récolter des informations sur les dépôts réels sur plusieurs décennies. Nous avons utilisé des techniques d'analyse stratigraphique et de datation des dépôts $\left({ }^{137} \mathrm{Cs},{ }^{210} \mathrm{~Pb}\right.$, géochimie) pour évaluer la hauteur atteinte par les eaux lors de différents évènements d'inondation, et proposer une chronologie des crues de la Medjerda. L'analyse pluridisciplinaire de ces archives sédimentaires permet de fournir de précieuses informations sur l'évolution climatique des dernières décennies, les événements extrêmes et enfin sur les effets des actions anthropiques passées et récentes (Vilanova et al., 2006 ; Selby et Smith, 2007).

\section{Matériel et méthode}

\subsection{Site d'étude du bassin versant de la Medjerda}

Le fleuve Medjerda est le plus long de Tunisie, avec $484 \mathrm{~km}$ dont $350 \mathrm{~km}$ en Tunisie (Ben Mansoura et al., 2001). Il prend sa source près de Souk-Ahras, dans le Constantinois algérien, puis coule vers l'est avant de se jeter dans la mer Méditerranée dans le golfe de Tunis. La Medjerda draine $23600 \mathrm{~km}^{2}$ dont $7500 \mathrm{~km}^{2}$ en Algérie (Habaieb, 1992) (Fig. 1).

Avant de se jeter dans la mer, la Medjerda traverse trois types de plaines :

- en amont, la haute vallée (du côté de Ghardimaou) est constituée de bassins colmatés par des sédiments quaternaires. Le fleuve et ses affluents sont caractérisés par des méandres encaissés de 8 à $10 \mathrm{~m}$. Ces régions appartiennent à des zones faiblement montagneuses. Les fortes crues ne débordent qu'exceptionnellement en dehors du lit du fleuve (Kotti et al., 2016) ;

- en aval de la retenue de Sidi Salem, de Testour à Jdaida, dite moyenne vallée de la Medjerda, le fleuve est encaissé dans la plaine. L'encaissement repasse à plus de $10 \mathrm{~m}$ en aval de Laroussia avant de s'atténuer rapidement vers Jdaida. Dans tout ce secteur la plaine est inondable surtout dans la partie centrale. En allant vers la mer les reliefs de ce bassin s'adoucissent ;

- dans la basse plaine, en aval de Jdaida, commence le delta. La pente de la Medjerda est encore marquée et son tracé a fréquemment été modifié, soit naturellement soit par des travaux (coupure de méandres, ouverture de nouvel émissaire...). Du point de vue géologique, la Medjerda est occupée par des alluvions quaternaires le plus souvent argilo-sableuses à argilo-sablolimoneuses.

La pluviométrie moyenne annuelle dépasse $1000 \mathrm{~mm}$ au nord du bassin versant de la Medjerda et diminue progressivement vers le sud. Il s'agit d'une pluviométrie souvent concentrée dans le temps, irrégulière et inégalement répartie sur les différentes saisons (Kotti et al., 2016). La base des données hydrologique provient en grande partie de la Direction Générale 


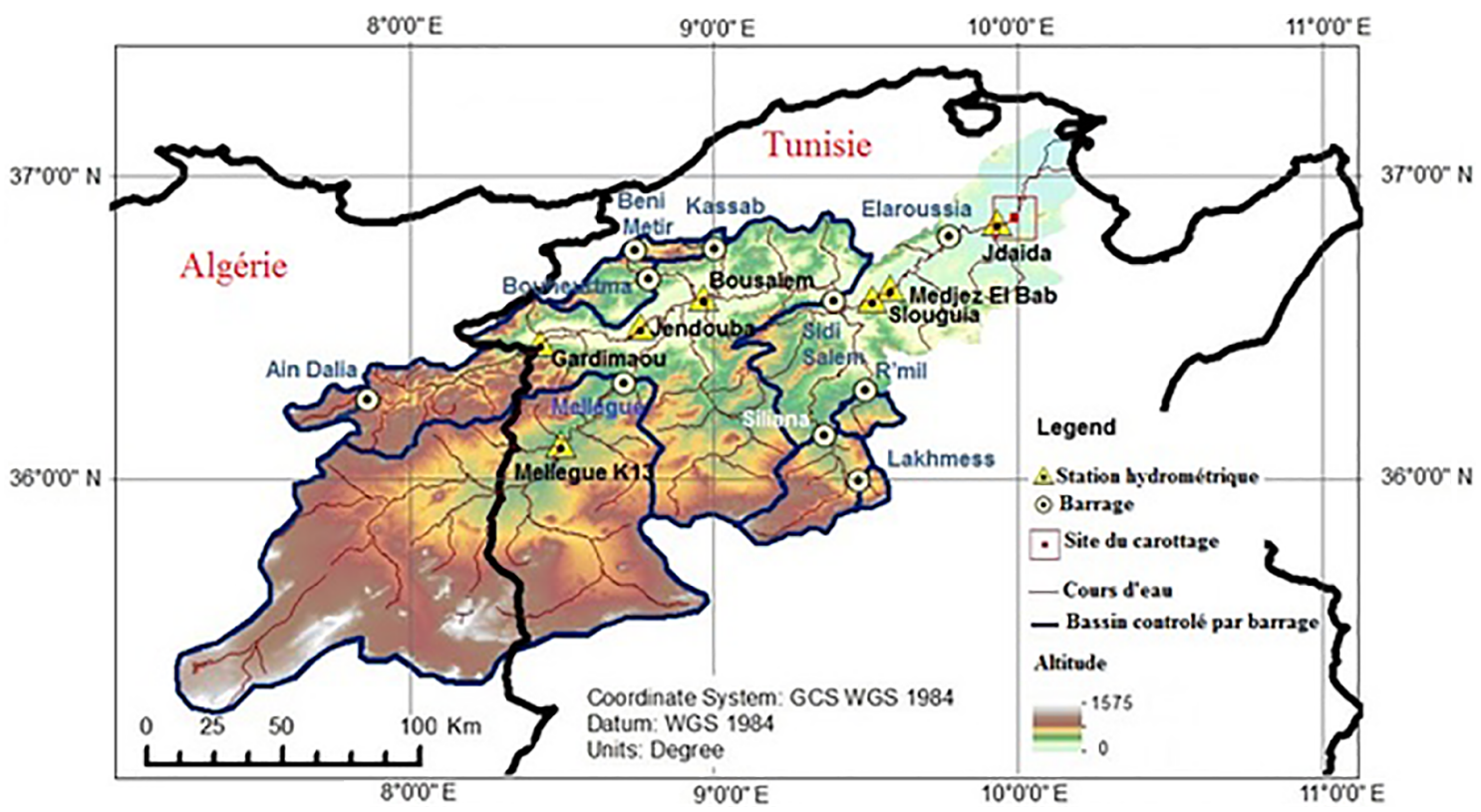

Figure 1. Localisation des grandes stations hydrométriques et des barrages sur le bassin versant de la Medjerda, et site du carottage.

des Ressources en Eau. Par ailleurs le laboratoire HydroSciences Montpellier (HSM) dispose d'une base de données pluviométrique importante au pas d'espace du demi-degré carré, qui propose des valeurs mensuelles de pluie par maille de $2500 \mathrm{~km}^{2}$ sur la période 1940-1999, complétée par des données nationales (DGRE).

\subsection{Régime hydrologique et stratégie de mobilisation des eaux de surface}

Le régime hydrologique est typiquement méditerranéen. Les contrastes saisonniers sont très marqués puisque, pendant l'été, le débit d'étiage peut se réduire à moins d' $1 \mathrm{~m}^{3} \mathrm{~s}^{-1}$ tandis que, pendant le reste de l'année, celui d'une crue de périodicité décennale atteint $1000-1200 \mathrm{~m}^{3} \mathrm{~s}^{-1}$ (Rodier et al., 1981). Le régime hydrologique de ce fleuve très hétérogène $\mathrm{a}$ été régulé par de nombreux ouvrages construits sur son cours principal ou ses affluents. A partir de 1945, un programme d'équipement de la Medjerda a été entrepris par la Direction des Travaux Publics de Tunisie, destiné à mettre en valeur les ressources encore inutilisées de cette vallée. Ce programme a permis la construction du barrage régulateur de Mellegue et la construction du barrage de Beni Metir (Strugo, 1955). Depuis l'indépendance de la Tunisie, le programme de développement du bassin de la Medjerda a été poursuivi grâce à la réalisation de plusieurs ouvrages : Lakhmes (1966), Kasseb (1968), Bouhertma (1976), Siliana (1987), Sidi Salem (1981) et R'Mil (1999). En Algérie, un seul grand barrage a été identifié, Ain Dalia, mis en eau en 1988 et d'une capacité de $82 \mathrm{Mm}^{3}$. Avec la mise en eau de ces barrages, le régime hydrologique du fleuve Medjerda a été modifié (Fig. 1 pour la localisation et tableau 1).

\subsection{Technique de prélèvements des sédiments, méthodes de mesure et géochronologie}

Le site du carottage a été choisi après plusieurs missions de terrain $(2013,2014,2015)$ qui avaient pour objectif d'identifier différents sites potentiellement intéressants pour la thématique. Le site d'étude est localisé dans un méandre en aval du dernier barrage -Sidi Salem- mais en amont de la zone estuarienne, proche de la station hydrologique Jdaida, la dernière avant la mer. Trois carottes ont été échantillonnées sur cette même terrasse alluviale, $\mathrm{C} 1 \mathrm{de} 90 \mathrm{~cm}, \mathrm{C} 3 \mathrm{de} 120 \mathrm{~cm}$ et C5 de $300 \mathrm{~cm}$ de profondeur. L'approche paléohydrologique a été utilisée pour comparer les résultats sédimentologiques et géochronologiques de cette archive sédimentaire aux informations de la station hydrométrique de Jdaida située juste en amont du site d'étude choisi.

L'identification et la mise en évidence des différents événements de crues ont été réalisées grâce à l'inspection minutieuse de chaque dépôt (décompte des lamines, identification de paléosols, détection des surfaces d'érosion, étude de la variabilité granulométrique à partir d'un granulomètre laser Beckman Coulter LS 13320 au laboratoire Géosciences Montpellier.

Toute reconstitution paléo-environnementale, à partir de l'étude des archives sédimentaires, nécessite l'établissement d'une échelle chronologique la plus fine possible, afin de dater avec une bonne résolution les événements hydrologiques passés. Les isotopes principalement utilisés pour dater les événements du siècle dernier sont le ${ }^{210} \mathrm{~Pb}$ et le ${ }^{137} \mathrm{Cs}$ (Dezileau et al., 2014a). Ils permettent une datation relativement précise, grâce à leurs propriétés chimiques (réactivité visà-vis des particules) et physiques (périodes de désintégra- 
Tableau 1. Caractéristiques des aménagements construits sur le bassin versant de la Medjerda (DGBGTH, 2016).

\begin{tabular}{|c|c|c|c|c|c|}
\hline $\begin{array}{l}\text { BARRAGES/ } \\
\text { Mise en eau }\end{array}$ & $\begin{array}{r}\text { Superficie } \\
\text { du bassin } \\
\left(\mathrm{km}^{2}\right)\end{array}$ & $\begin{array}{r}\text { Capacité } \\
\text { totale initiale } \\
\left(\mathrm{Mm}^{3}\right)\end{array}$ & $\begin{array}{r}\text { Envasement des } \\
\text { retenues Mesurée } \\
\left(\mathrm{Mm}^{3}\right)\end{array}$ & $\begin{array}{r}\text { Taux } \\
\text { d'envasement des } \\
\text { retenues }(\%)\end{array}$ & $\begin{array}{r}\text { Taux d'envasement } \\
\text { annuel des retenues } \\
(\%)\end{array}$ \\
\hline MELLEGUE (1954) & 10300 & 182 & 122 & 67 & 1,36 \\
\hline BENI METIR (1954) & 103 & 62 & 1 & $*$ & * \\
\hline KASSEB (1966) & 170 & 81 & 5 & 6,08 & 0,19 \\
\hline SIDI SALEM (1981) & 17885 & 814 & 171 & 21 & 0,86 \\
\hline BOU HEURTMA (1976) & 457 & 117 & 5 & 4,65 & 0,16 \\
\hline SILIANA (1987) & 972 & 70 & 37 & 52,2 & 3,25 \\
\hline LAKHMESS (1968) & 127 & 8,22 & 1 & 12,2 & 0,37 \\
\hline RMIL (1999) & 221 & 4 & $*$ & * & $*$ \\
\hline AIN DALIA (1988) & 193 & 82 & (64) & (22) & 0,91 (ANBT) \\
\hline
\end{tabular}

* Données manquantes.

tion, rayonnement gamma). Le ${ }^{137} \mathrm{Cs}$ et le ${ }^{210} \mathrm{~Pb}$ sont mesurés par spectrométrie gamma (détecteur CANBERRA BEGe 3825) au laboratoire Géosciences Montpellier (France) et au CNSTN à Sidi Thabet (Tunisie).

$$
\text { - Datation par le }{ }^{210} \mathrm{Pbex}(\mathrm{T} 1 / 2=22,3 \text { ans })
$$

Le Plomb 210 est un isotope radioactif naturel issu de la chaîne de désintégration de l'Uranium 238. L'Uranium 238, contenu naturellement dans la croûte terrestre, subit la série de désintégration suivante : Uranium 238 - Uranium 234 - Thorium 230 - Radium 226 - Radon 222. Le $222 \mathrm{Rn}(\mathrm{T} 1 / 2=3,8$ jours $)$ ainsi produit, se trouve sous une forme gazeuse. Lorsqu'il est produit à proximité de la surface, le gaz s'échappe dans l'atmosphère où il se transforme rapidement en Polonium 218 qui se désintègre en quelques minutes en ${ }^{210} \mathrm{~Pb}$. Ce ${ }^{210} \mathrm{~Pb}$, dit atmosphérique (ou en excès) retombe sous forme de particules humides ou sèches et s'accumule dans les sédiments. Son activité au sein de la colonne sédimentaire diminue avec le temps selon une loi de décroissance radioactive. Le principe de la datation au ${ }^{210} \mathrm{~Pb}$ s'appuie sur l'hypothèse selon laquelle le flux atmosphérique en ${ }^{210} \mathrm{~Pb}$ est constant, ce qui implique que l'activité de l'isotope radioactif à la surface des sédiments reste toujours identique. Cette hypothèse permet en mesurant l'activité $\mathrm{du}{ }^{210} \mathrm{~Pb}$ et en appliquant la loi de décroissance radioactive de déterminer l'âge de nos sédiments. Au bout de cinq fois sa période, c'est à dire 110 ans, il a complètement disparu dans l'environnement (Dezileau et al., 2014b). La mesure du ${ }^{210} \mathrm{~Pb}$ sur le détecteur gamma CANBERRA se fait en intégrant le pic à $46,5 \mathrm{keV}$. Le ${ }^{210} \mathrm{Pbex}$ est estimé en soustrayant le pic $\mathrm{du}^{210} \mathrm{~Pb}$ au pic du ${ }^{226} \mathrm{Ra}$ à $186,2 \mathrm{keV}$. L'erreur n'excède pas $6 \%$.

- Datation par le ${ }^{137} \mathrm{Cs}(\mathrm{T} 1 / 2=30,17$ ans $)$

Le Césium 137 est un isotope radioactif artificiel issu de réactions de fission nucléaire. Il est relâché dans l'atmosphère lors de l'utilisation de bombes atomiques et d'accidents dans des centrales nucléaires. Avec une période de demi-vie $\left(30,17\right.$ ans) proche de celle du ${ }^{210} \mathrm{~Pb}$, il permet de dater les séquences sédimentaires sur une même échelle temporelle. Les profils d'activité du Césium 137 en fonction de la profondeur dans une coupe sédimentaire présentent des pics dont le plus important est généralement attribué à l'année 1963, date du maximum de rejets atmosphériques par les essais nucléaires. La présence de Césium 137 dans les dépôts sédimentaires indiquera qu'ils ont été déposés postérieurement aux années 1950. La mesure du ${ }^{137}$ Cs sur le détecteur gamma CANBERRA se fait en intégrant le pic à $661 \mathrm{keV}$. L'erreur n'excède pas $6 \%$.

\section{Résultats}

\subsection{Sédimentation des réservoirs}

Le taux d'envasement de chaque barrage construit sur le bassin versant de la Medjerda met en évidence l'importance de l'alluvionnement durant la durée de l'exploitation des retenues des barrages, dont Mellegue, Siliana et Sidi Salem qui sont sujettes à un alluvionnement plus important (tableau 1). Il a été estimé que le volume initial de la retenue de Sidi Salem sera envasé jusqu'à $60 \% 100$ ans après la construction du barrage, et l'envasement devrait être total pour les retenues des deux barrages de Mellegue et Siliana dans 60 à 65 ans (Ben Mammou et Louati, 2007). Des soutirages efficaces permettent dans ce cas de réduire le taux d'alluvionnement et d'augmenter la durée de vie de ces barrages. D'après Abid (1980), 59 à $64 \%$ des apports solides dans la retenue du barrage de Mellegue, durant la période 1954-1980, ont été soutirés grâce aux manœuvres de dévasement pendant les crues. La Fig. 2 montre le volume de vase évacué suite à des événements exceptionnels entre 1954 et 2003 au niveau du barrage Mellegue et entre 1982 et 2005 au niveau du barrage Sidi Salem. En 1969, de très importants volumes de sédiments évacués lors d'une crue se sont accumulés surtout dans les zones où la pente diminue, ainsi que dans les secteurs évasés. En mars 1973, l'oued Medjerda a connu une autre 

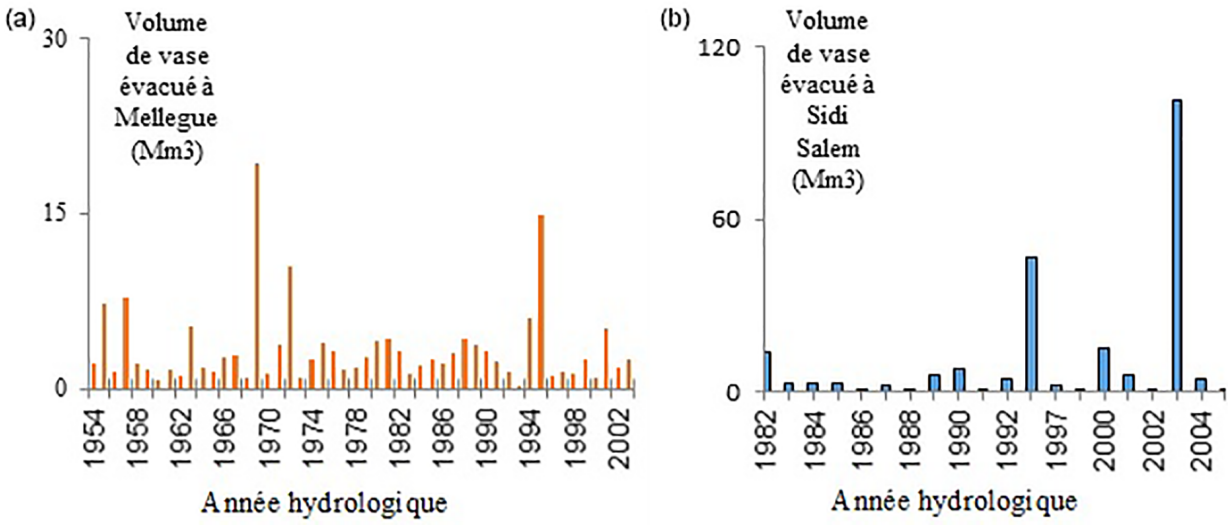

Figure 2. (a) Le volume de vase évacué au niveau du barrage Mellegue entre 1954 et 2003. (b) Le volume de vase évacué au niveau du barrage Sidi Salem entre 1982 et 2005.

crue plus intense provoquant de graves inondations avec une quantité exceptionnelle de matériaux transportée et ce malgré les gros travaux d'endiguement effectués à Jdaida (entre 1947 et 1957) (Ben Hassine and Rejeb, 2003).

Depuis la mise en eau du barrage Sidi Salem en 1981 jusqu'en 1990, le volume de vase évacué par ce barrage est quasiment nul. On remarque le faible pourcentage de vase évacuée dû à l'arrêt des opérations de soutirage. Quelques opérations de dévasement occasionnel ont été associées à des débits maximum exceptionnels : $595 \mathrm{~m}^{3} \mathrm{~s}^{-1}$ en 1990 , $671 \mathrm{~m}^{3} \mathrm{~s}^{-1}$ en $1995,977 \mathrm{~m}^{3} \mathrm{~s}^{-1}$ en 2000 et $1020 \mathrm{~m}^{3} \mathrm{~s}^{-1}$ 2003. Durant l'année 1995, les gestionnaires de Sidi Salem ont libéré un volume total dévasé de $24,27 \mathrm{Mm}^{3}$. Les particules solides se déposent en aval en fonction de la réduction de la vitesse du courant de turbidité, ainsi plusieurs facteurs peuvent influencer les épaisseurs des dépôts accumulés tels que la vitesse du courant, la topographie, la présence d'obstacles perturbant les dépôts, etc. Ces facteurs créent une variabilité latérale de dépôt importante, en aval de la retenue. Le lit de la Medjerda connaît de ce fait un engraissement continu depuis 1981, date de la mise en service du barrage Sidi Salem. De ce fait, et par manque de débits de crue important qui " chassent" les dépôts précédents, le débit occasionnant le débordement a diminué à moins de $200 \mathrm{~m}^{3} \mathrm{~s}^{-1}$, valeur nettement inférieure au débit de débordement avant la construction du barrage qui était de $700 \mathrm{~m}^{3} \mathrm{~s}^{-1}$.

La Fig. 2a illustre parfaitement l'accroissement des volumes solides évacués suite à la crue exceptionnelle de 2003. Quatre fortes crues très rapprochées sont survenues dans le bassin de la Medjerda au début de l'année 2003, après quatre années de sécheresse (Abdelhamid et Khalil, 2009). Elles ont cumulé un milliard de $\mathrm{m}^{3}$ d'apports à Sidi Salem en un mois. La retenue a été assez vite remplie en début d'hiver. Ainsi, après complet encaissement de la première crue dans le creux disponible, et déversement de la seconde crue, la troisième a poussé le déversement sur la tour et réduit la tranche de laminage résiduelle (Daoud et al., 2009).

\subsection{Modélisation et reconstitution des transports solides}

L'objectif principal de cette section est de développer un modèle simple qui montre l'atténuation du signal des apports solides à Jdaida avec les données disponibles sans tenir compte des paramètres physiques du bassin. La méthodologie s'intéresse d'abord au premier barrage Mellegue construit puisque, on dispose à cette station de 1954 à 2005 d'un historique des apports solides annuels au barrage MES $X_{\text {Mellegue }}$ et des volumes annuels de vase évacués MES lachées $Y_{\text {Mellegue. A la }}$ construction de chaque barrage correspond un stockage de MES que l'on peut dans un premier temps estimer par un pourcentage $Y_{n}$ d'une quantité de MES $X_{n}$. Le coefficient annuel de réduction de transport solide pour le barrage de Mellegue qui a été calculé est de l'ordre $51 \%$. Le fonctionnement sera étudié après pour tout le reste du bassin versant Medjerda, grâce à des valeurs calculées pondérées par la surface de chaque barrage en appliquant le même coefficient de réduction à Mellegue pour les autres barrages, ce qui constitue bien sur une hypothèse réductrice, mais basée sur la seule observation disponible.

Avant la mise en eau du barrage Sidi Salem en 1981, les barrages Mellegue, Beni Metir, Bouherma, Kassab et Lakhemes (tableau 2) déversaient vers la mer sans autre obstacle. Depuis 1981, les quantités de MES qui ressortent de ces barrages arrivent au barrage d'ordre 2 Sidi Salem, situé juste après la station Bousalem, sur le cours de la Medjerda (tableau 2). Depuis la mise en eau du barrage Siliana en 1987, les quantités qui ressortent du barrage Lakhemes arrivent au barrage Siliana d'ordre 2. Cette étude permet de classer l'ordre de grandeur des flux des matières solides jugés assez proche de la réalité. La quantité de MES qui arrive à Jdaida est calculée en additionnant les quantités de MES lâchées par chaque barrage avec les quantités de MES produites dans le bassin intermédiaire, en appliquant à chaque fois la réduction de MES déjà calculée à Mellegue. Les résultats de cette approch, illustrés par la Fig. 3 et le tableau 2, montrent la réduction de la quantité de MES calculé à la sta- 


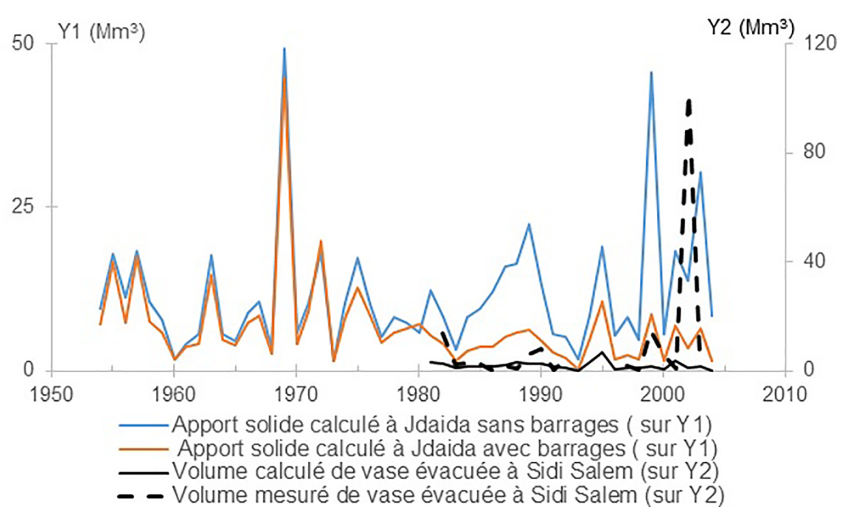

Figure 3. Evolution des apports solides calculés à Jdaida et des volumes calculés et mesurés de vase évacuée à Sidi Salem.

tion de Jdaida depuis la mise en eau du barrage Sidi Salem. Notons tout de même qu'il faut prendre les résultats obtenus avec beaucoup de précaution, cependant cela produit un signal d'atténuation des apports solides qui peut servir à interpréter les carottes prélevées dans la basse vallée. Nous avons eu recours à la modélisation à l'aide d'un modèle simplifié pour pallier au manque des données réelles dans le but de l'estimation du transport solide dans la Medjerda, mais il faudrait mettre en place un programme de mesures de débits solides au sortir des barrages pour valider ce modèle. Cette approche doit être approfondie en prenant en compte les informations qui pourront être obtenues auprès des gestionnaires de barrages, afin de mieux identifier la variabilité locale de la production de MES et des taux de sédimentation dans les barrages. D'autres modèles seront peut-être mieux appropriés avec beaucoup plus de précision concernant entre autre la production de sédiments. Il est recommandable de donner plus d'effort et d'investissements de la part des organismes concernés pour pouvoir donner une estimation exacte du transport solide.

\subsection{Approche paléohydrologique pour évaluer l'impact anthropique}

\subsubsection{Reconstitution de la variabilité passée des transports sédimentaires du bassin versant de la Medjerda, Tunisie}

L'utilisation de données granulométriques est indispensable à l'étude des archives sédimentaires. On se limite dans cette partie au résultat d'une seule carotte $\mathrm{C} 5$, la plus profonde et la plus riche en couches de sédiments différentes à interpréter. Les deux autres carottes $\mathrm{C} 1$ et $\mathrm{C} 3$ ne sont pas assez profondes pour atteindre le pic de Cesium, mais elles mettent en évidence une certaine variabilité latérale des dépôts, tout en confirmant la nature essentiellement argileuse des premiers $80 \mathrm{~cm}$ de dépôt.

La mise en place d'un log litho stratigraphique nous a permis d'identifier visuellement les différents dépôts de crues.
L'archive sédimentaire montre d'importantes variations granulométriques en fonction de la profondeur, d'un matériel silteux-argileux à sableux (D50 compris entre 5 et $240 \mu \mathrm{m})$. Ces niveaux sableux sont d'un grain fin à moyen (de 63 à $449 \mu \mathrm{m}$ ) aux niveaux des profondeurs $125 \mathrm{~cm}, 150,210,250$ et $290 \mathrm{~cm}$, indiquant un régime hydraulique plus énergétique. Ces pics correspondent probablement à des événements exceptionnels (crues intenses).

L'ensemble des travaux d'observation, des études granulométriques et géochimiques de la carotte C5 permet d'identifier de manière plus fine le nombre de dépôts de crues. Les variations granulométriques et géochimiques sont le reflet des modifications de la dynamique sédimentaire. Le nombre de dépôts de crue est estimé à 18 et sont notés de FD1 à la base de l'archive à FD18 au sommet. Dans la séquence des dépôts de crue de la carotte C5, l'unité de dépôt FD1 présente une activité non négligeable de ${ }^{137} \mathrm{Cs}$ (Fig. 4) indiquant que tous les dépôts de crue de FD1 à FD18 sont tous postérieurs à 1950. D'autre part, la forte activité en cesium-137 mesurée au niveau de l'unité FD 7 (172 cm; 8,2 $\left.\mathrm{mBqg}^{-1}\right)$, nous permet de faire la supposition que ce dépôt de crue est associé à la période de production maximale de Cesium-137 dans l'atmosphère, c'est à dire au milieu des années 1960 (autour de 1963, Fig. 4). Le ${ }^{210} \mathrm{~Pb}$ est en excès dans l'unité FD1, ce qui indique que les dépôts de crue FD1-FD18 sont récents et tous postérieurs aux années 1900. D'autre part, nous avons trouvé sur le terrasse alluviale entre 120 et $140 \mathrm{~cm}$, des déchets de pots de yaourth "Sami" fabriqué dans les années 1970.

Ces traceurs chronologiques, nous ont permis de mettre en place une chronologie approximative des dépôts de crue (FD1 à FD18) que nous comparons à l'historique des crues disponibles depuis 1930 à la station de Jdaida (Fig. 4). La comparaison de ces enregistrements permet d'attribuer de manière plus fine des âges aux dépôts de crues de la séquence FD. L'événement de 1969, le premier événement puissant après 1963 enregistré par les archives instrumentales $\left(832 \mathrm{~m}^{3} \mathrm{~s}^{-1}\right)$, a probablement déposé une unité de texture différente en silt limite sables fins (FD8 : $44 \mu \mathrm{m})$. Un autre événement plus puissant encore, est enregistré en 1973 avec un débit estimé à $3500 \mathrm{~m}^{3} \mathrm{~s}^{-1}$ à Medjez El Bab. Les mesures hydrologiques réalisées pendant la crue montrent qu'un débit de $850 \mathrm{~m}^{3} \mathrm{~s}^{-1}$ est passé dans le lit du fleuve, mais que le reste a débordé déclenchant des inondations exceptionnelles (Claude et al., 1977). Si nous supposons qu'il existe une corrélation entre l'intensité d'un événement et la taille granulométrique qui caractérise son dépôt (Dezileau et al., 2014b), alors nous pourrions associer l'événement de 1973 à l'unité FD10 caractérisée par des sables fins (FD10 : $59 \mu \mathrm{m}$, $20 \mathrm{~cm}$ d'épaisseur). Cela est cohérent par rapport aux datations au ${ }^{137} \mathrm{Cs}$ et la découverte dans ces dépôts de pots de yaourt "Sami ". En nous fondant sur une relation possible entre la granulométrie des sédiments et l'ampleur d'un événement, FD17 (FD $7: 100 \mu \mathrm{m})$ pourrait être associé à 2003, pour un débit $750 \mathrm{~m}^{3} \mathrm{~s}^{-1}$ à Jdaida et FD 12 avec du maté- 
Tableau 2. Rapport sur valeur moyenne de MES calculé au niveau de chaque barrage et des valeurs moyennes de MES calculées à Jdaida.

\begin{tabular}{|c|c|c|c|}
\hline \multirow[b]{2}{*}{ Barrage } & \multirow[b]{2}{*}{$\begin{array}{l}\text { Ordre de grandeur } \\
\text { des flux des } \\
\text { des matières solides }\end{array}$} & \multicolumn{2}{|c|}{ valeur moyenne de MES calculé $\left(\mathrm{m}^{3}\right)$} \\
\hline & & $\begin{array}{r}\text { stockée dans le } \\
\text { barrage }\end{array}$ & $\begin{array}{r}\text { lâchée par le } \\
\text { barrage }\end{array}$ \\
\hline Mellegue & Barrage d'ordre 1 & 2136732 & 3269028 \\
\hline Beni Metir & Barrage d'ordre 1 & 21359 & 32677 \\
\hline Lakhemes & Barrage d'ordre 1 & 28770 & 42291 \\
\hline Kassab & Barrage d'ordre 1 & 38070 & 56020 \\
\hline Bouhertma & Barrage d'ordre 1 & 131856 & 144417 \\
\hline Sidi Salem & Barrage d'ordre 2 & 2169357 & 1901540 \\
\hline Siliana & Barrage d'ordre 2 & 300445 & 258208 \\
\hline Passant à Jdaida & avec les barrages & 6718661 & \\
\hline Passant à Jdaida & sans les barrages & 11503176 & \\
\hline
\end{tabular}
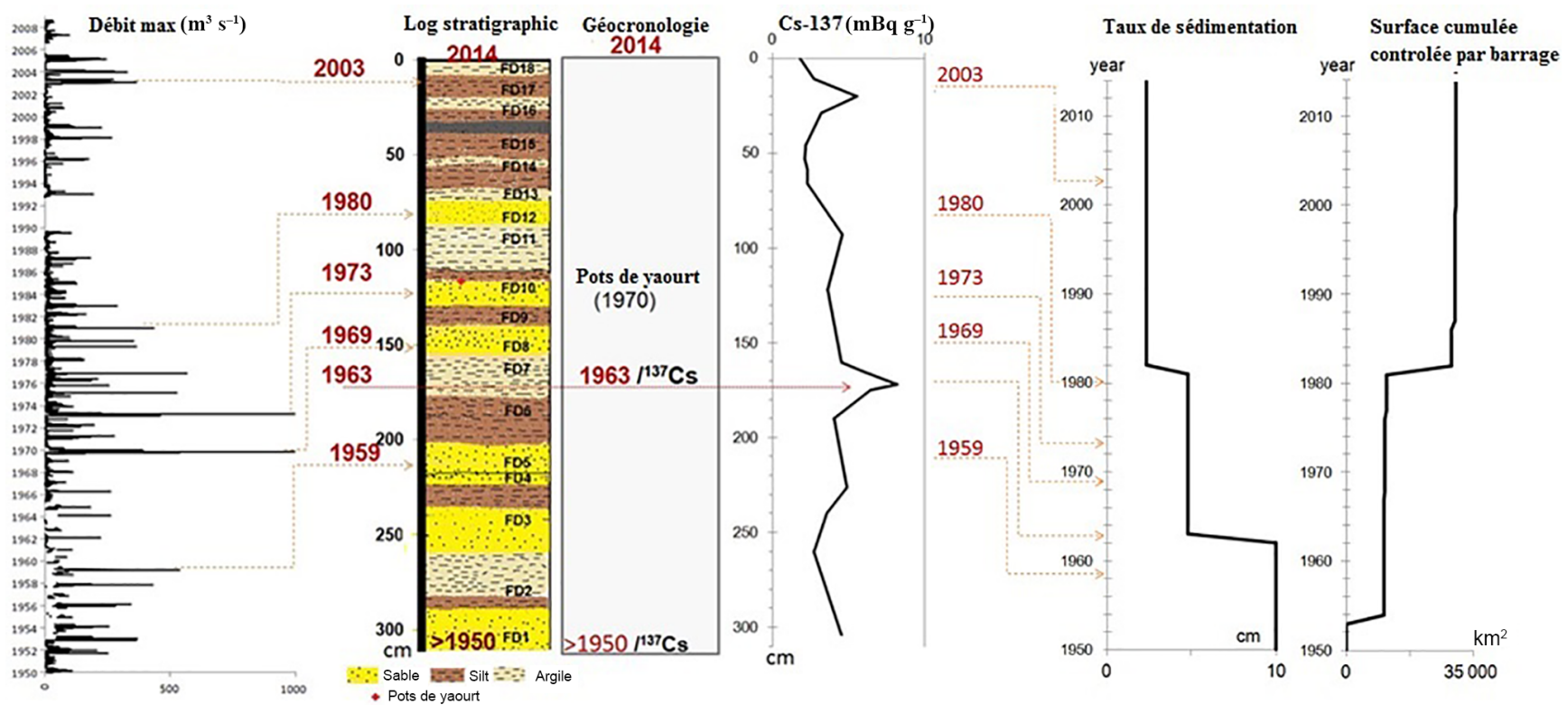

Figure 4. Reconstitution historique des apports sédimentaire par les traceurs chronologiques $\left({ }^{137} \mathrm{Cs}\right)$ et la comparaison des enregistrements de débit maximum annuel au niveau de la station hydrométrique de Jdaida.

riel fin (D50 of $26 \mu \mathrm{m}$ ) à 1980 (Fig. 4). D'autres unités sousjacentes FD5 avec D50 de $212 \mu \mathrm{m}$, FD4 avec D50 de $212 \mu \mathrm{m}$ et FD1 avec D50 de $224 \mu \mathrm{m}$ ont été affectées à quatre événements de plus faible ampleur que celui de 1973 (538, 432 et $344 \mathrm{~m}^{3} \mathrm{~s}^{-1}$ ) qui ont eu lieu en 1959, 1957 et 1956 (ou 1952) respectivement (Fig. 4). Les couches sédimentaires associées à ces crues sont caractérisées par des sables moyens (211 à $228 \mu \mathrm{m})$. Ceci est expliqué par le fait qu'avant 1969, les sections du cours d'eau étaient dégagées, la terrasse alluviale de la zone d'étude était en évolution. Cette approche nous a permis de tracer l'histoire sédimentaire à travers l'étude de la carotte de sédiments prélevés. Les résultats indiquent un dépôt de $75 \mathrm{~cm}$ entre 1981 et 2014 , alors qu'il était de $230 \mathrm{~cm}$ entre 1956 (ou 1952) et 1981 (120 cm entre 1956/52 et 1963, puis $105 \mathrm{~cm}$ entre 1963 et 1981). L'histoire sédimentaire du fleuve Medjerda montre une réduction du flux sédimentaire à l'aval du barrage de Sidi Salem du fait des multiples aménagements.

\subsubsection{Etude de l'évolution des apports : impact anthropique}

La plaine de Medjerda est l'une des zones les plus peuplées de Tunisie, 288834 habitants en 2004 (INS). Depuis l'indépendance en 1956, le pays a connu une croissance rapide de la population, et les zones urbaines au nord ont augmenté de 7,16\% entre 1950 et 2007 (Samaali, 2011). Compte tenu de l'augmentation des utilisations de l'eau dans les pôles économiques urbains au nord-est et au centre-est, l'état a suivi un plan de développement pour la mise en place d'un grand 
Tableau 3. Evolution des sédiments transportés dans la basse vallée de Medjerda depuis 1950.

\begin{tabular}{|c|c|c|c|}
\hline Année & $1950-1963$ & $1963-1981$ & 1981-2014 \\
\hline Nombre de grands barrages & 2 & 5 & 9 \\
\hline Surface cumulée contrôlée $\mathrm{km}^{2}$ & 10304 & 11161 & 30445 \\
\hline Taux de transport sédimentaire $\mathrm{cm}^{-1}$ & 10 & 4,75 & 2,34 \\
\hline Nature de sédiment & $\begin{array}{r}\text { sable fin et } \\
\text { sable grossier }\end{array}$ & silt et sable fin & silt ou argile \\
\hline
\end{tabular}

nombre de retenues, dont 9 grands barrages construits dans le bassin versant de la Medjerda, afin de répondre à l'intérêt général.

Les résultats d'analyse granulométrique de la carotte C5 montrent principalement une succession de petites couches de matériel fin (silt ou argile) depuis la mise en eau du barrage Sidi Salem en 1981 et un taux de sédimentation faible $\left(2,3 \mathrm{~cm} \mathrm{an}^{-1}\right)$, alors qu'il était beaucoup plus important, environ $4.75 \mathrm{~cm} \mathrm{an}^{-1}$ entre 1963 et 1981 , et environ $10 \mathrm{~cm} \mathrm{an}^{-1}$ entre 1950 et 1963 (tableau 3).

Ceci illustre bien l'impact de la mise en eau des nombreux barrages sur le bassin, qui prive la basse vallée, l'estuaire et la zone littorale de la majeure partie des apports sédimentaires, en particulier grossiers.

\section{Discussion}

Généralement, les barrages limitent les apports de sédiments à la mer et participent au déficit général de sédiments sur les côtes. Ils capturent jusqu'à $30 \%$ des flux de sédiments produits sur les massifs (Vorosmarty et al., 2003). Depuis la construction des barrages sur le bassin versant de la Medjerda, dont le plus important, celui de Sidi Salem, le lit de la Medjerda a connu d'importants changements morphologiques dans sa basse vallée, et en particulier un important engraissement du lit mineur. Cet engraissement est dû en grande partie à la réduction des débits de crue exceptionnels qui devraient " chasser" les dépôts anciens du lit mineur. Ceci a diminué la capacité de transit du fleuve ainsi que la vitesse d'écoulement entre la sortie du barrage et l'estuaire. Il en résulte l'apparition de débordements plus fréquents et la propagation des inondations pour des débits de plus en plus faibles. Par ailleurs, les crues ont également beaucoup moins d'impact mécanique sur la région côtière.

Les résultats de mesures de l'alluvionnement menées depuis une vingtaine d'année, montrent l'importance de la sédimentation dans les retenues des barrages de Tunisie. Des concentrations supérieures à $100 \mathrm{~g} \mathrm{~L}^{-1}$ ont été enregistrées dans les eaux lors des crues de la Medjerda (Rodier et al., 1981). De ce fait, les retenues créées par les différents ouvrages hydrauliques sont toutes confrontées à plus ou moins long terme à l'alluvionnement. Les mesures de transport solide effectuées en Tunisie et les études ponctuelles détaillées restent insuffisantes et n'ont pas été menées assez longtemps pour permettre des études exhaustives sur le sujet. La modélisation du transport sédimentaire proposée dans cette étude résulte donc d'une approche simplifiée destinée à montrer l'atténuation du signal des apports sédimentaires, calculé à partir de données fragmentaires de transport solide et de taux d'envasement disponibles sur quelques sites d'étude sur le bassin. Cette première approche indique une réduction croissante des transports sédimentaires avec l'augmentation du nombre de barrages, qui peut servir également à supporter l'interprétation des archives sédimentaires prélevées dans les carottes de la basse vallée.

\section{Conclusion}

La Medjerda est devenu un fleuve presque totalement contrôlé, et les matières solides qu'il apporte ont été réduites de façon considérable. Ce qui indique aussi que les crues ont beaucoup moins d'impact mécanique sur la région côtière. La mise en évidence de différents paléo-événements de crue qui ont été datés par des méthodes complémentaires $\left({ }^{137} \mathrm{Cs}\right.$, ${ }^{210} \mathrm{Pbex}$, géochimie, historiques des crues) montre que la terrasse FD a enregistré l'ensemble des événements de crue entre 1952 (ou 1956) et 2014. Une succession visible de couches sédimentaires correspondant aux dépôts des différentes crues qui se sont succédées sur le site d'étude a été déterminée. Elle permet de reconstituer l'histoire des apports sédimentaires du fleuve Medjerda jusqu'à la mer sur une période de 60 ans, avant et après la construction de nombreux barrages. Cette approche a permis d'évaluer le taux de réduction et la variabilité interannuelle des transports des sédiments. Toutefois, il faudrait une étude multi-site pour valider cette approche.

D'après la carotte $\mathrm{C} 5$, le taux de sédimentation était d'environ $10 \mathrm{~cm} \mathrm{an}^{-1}$ entre $1952 / 56$ et 1963 , principalement constitue de sables, puis il est passé à environ $4.75 \mathrm{~cm} \mathrm{an}^{-1}$ entre 1963 et 1982, avec un équilibre plus net entre sables, silts et argiles. Après la mise en eau du barrage Sidi Salem en 1981 sur le cours d'eau principal, les apports consistaient principalement en matériel fin, avec un taux de sédimentation faible $\left(2,3 \mathrm{~cm} \mathrm{an}^{-1}\right)$, Il s'agit d'une diminution significative des apports sédimentaires à la mer, et d'une modification du type de sédiments, essentiellement fins avec une disparition du matériel grossier, dû aux barrages, et en particulier celui de Sidi Salem sur le fleuve Medjerda. Actuel- 
lement la surface contrôlée par les barrages existants sur le bassin de la Medjerda est de $130 \%$ de la surface du bassin de la Medjerda, ce qui veut dire que l'eau de ruissellement est stockée 1,3 fois sur son parcours vers l'estuaire, avec les conséquences enregistrées dans la carotte sur la dynamique sédimentaire pré-estuarienne. D'autres études (Benmoussa et al., 2017), illustrent l'impact de ce déficit d'apports sédimentaires dans la morphodynamique côtière du golfe de Tunis.

Disponibilité des données. Des données sont disponibles sur demande.

Intérêts concurrents. Les auteurs déclarent qu'ils n'ont acun conflit d'intérêts.

Déclaration de la numéro special. This article is part of the special issue "Water quality and sediment transport issues in surface water". It is a result of the IAHS Scientific Assembly 2017, Port Elizabeth, South Africa, 10-14 July 2017.

Remerciements. Nous remercions l'Institut National Agronomique de Tunisie et son Directeur Monsieur Mahmoud Elyes Hamza, pour le soutien logistique et financier, et pour l'octroi d'une bourse de stage à l'Université de Montpellier. Le programme Campus France est particulièrement remercié pour l'attribution d'une bourse Eiffel de séjour long en France, ainsi que l'IRD pour une bourse de séjour scientifique long en France. Nous remercions aussi le Centre National des Sciences et Technologies Nucléaires pour la disponibilité du matériel de carottage.

Edited by : Christophe Cudennec

Reviewed by : Didier Orange and one anonymous referee

\section{Références}

Abdelhamid, D. and Khalil, J. : Couplage d'un évacuateur vanné avec une tranche de laminage, cas du barrage de Sidi Salem en Tunisie, Colloque CFBR-SHF : "Dimensionnement et fonctionnement des évacuateurs de crues", 20-21 janvier, Lyon, 2009.

Abid, A. : Apports solides et soutirages aux barrages Nebeur sur l'oued Mellegue (1954-1980), Séminaire International d'Experts sur le Dévasement des retenues, Tunis, 1-4 juillet, 13 pp., 1980.

Baker, V. R., Webb, R. H., and House, P. K. : The scientific and societal value of paleoflood hydrology, in : Ancient Floods, Modern Hazards : Principles and Applications of Paleoflood Hydrology, edited by : House, P. K., Webb, R. H., Baker, V. R., Levish, D. R., Water Science and Application Series, 5, 127-146, 2002.

Bates, B. C., Kundzewicz, Z. W., Wu, S., and Palutikof, J. P. : Le changement limatique et l'eau, document technique publié par le Groupe d'experts intergouvernemental sur l'évolution du climat, Secrétariat du GIEC, Genève, 236 pp., 2008.

Ben Hassine, H. and Rejeb, M. M. : Crues et inondations dans la moyenne Mejerda, Rapport de synthèse DGRE, 2003.
Ben Mammou, A. and Louati, M. H. : Evolution temporelle de l'envasement des retenues de barrages Tunisie. Revue des sciences de l'eau, Journal of water science, 20, 201-210, 2007.

Ben Mansoura, A., Garchi, S., and Daly, H. : Analyzing forest users' destructive behavior in Northern Tunisia, Land Use Policy, 18, 153-163, 2001.

Benmoussa, T., Amrouni, O., Dezileau, L., Mahe, G., and Abdeljaouad, S. : Impact of the Medjerda sedimentary fluxes on the morphodynamic equilibrium of the northern coast of the Gulf of Tunis (Medjerda-Raoued coast), Tunisia, PIAHS 377, IAHS meeting, Port Elizabeth, South Africa, 2017.

Claude, J., Francillon, G., and Loyer, J. Y. : Les alluvions déposées par l'Oued Medjerda lors des crues exceptionnelles de Mars 1973, Cah. ORSTOM, sér. Hydrol., vol. XIV, no. I1, 1977.

Daoud, A. and Jemmali, K. : Couplage d'une vacuateur vanne avec une tranche de laminage, cas du barrage Sidi Salem en Tunisie Combining gated spillway and flood damping, Sidi Salem dam in Tunisia. Colloque CFBR-SHF : "Dimensionnement et fonctionnement des évacuateurs de crues", 20-21 janvier 2009, Lyon - Daoud Jemmali Goguel Leclerc, Couplage d'un évacuateur vanné avec une tranche de laminage, cas du barrage de Sidi Salem en Tunisie, 2009.

Dezileau, L., Terrier, B., Berger, J. F., Blanchemanche, P., Freydier, R., Bremond, L., Latapie, A., Paquier, A., Lang, M., and Delgado, J. L. : A multidating approach applied to historical slackwater flood deposits of the Gardon River, SE France, Geomorphology, 214, 56-68, https://doi.org/10.1016/j.geomorph.2014.03.017, 2014a.

Dezileau, L., Terrier, B., Berger, J. F., Blanchemanche, P., Latapie, Freydier, R., Paquier, A., Lang, M., and Delgado, J. L. : Paleohydrological reconstruction of extreme floods of the Gardon River, SE France, Houille Blanche, International Water Journal, 4, 4452, 2014b.

Ghorbel, A. and Claude, J. : Mesure de l'envasement dans les retenues de sept barrages en Tunisie : estimation des transports solides, DRES-ORSTOM, No. : 049 34, Tunis, 1982.

Habaieb, H. : Comparaison numérique des modèles de prévision des crues, application à des bassins versants belge, français et tunisien. Doctorat d'Etat en Sciences Agronomiques de l'Université de Gand, Belgique, Mai 1992.

Huntington, T. : Evidence for intensification of the global water cycle : Review and synthesis, J. Hydrol., 319, 83-95, 2006.

Kotti, F., Mahe, G., Habaieb, H., Dieulin, C., and Calvez, R. : Etude des pluies et des débits sur le bassin versant de la Medjerda, Tunisie, Bulletin de l'Institut Scientifique, 38, 19-28, 2016.

Labat, D., Godderis, Y., Probst, J., and Guyot, J. : Evidence for global runoff increase related to climate warming, Adv. Water Resour., 27, 631-642, 2004.

Rodier, J., Colombani, J., and Kallel, R. : Monographie de la Medjerda, ORSTOM, DGRE, 1981.

Samaali, H. : Etude de l'évolution de l'occupation et de l'utilisation du sol dans le delta de Medjerda par télédétection et systèmes et systèmes d'informations géographiques, Thèse en vue de l'obtention du Doctorat en Géographie. Université de Tunis Faculté des Sciences Humaines et Sociales Ecole Doctorale : Structures, Systèmes et Modèles en Lettres et Sciences Humaines, 2011.

Selby, K. A. and Smith, D. E. : Late Devensian and Holocene sealevel changes on the Isle of Skye, Scotland, UK, J. Quaternary Sci., 22, 119-139, 2007. 
Solomon, S. : Climate change 2007 : the physical science basis, Cambridge University Press Cambridge, 2007.

Strugo, N. : La mise en valeur de la valeur de la vallée de la Medjerda, BEST, 102, 49-95, 1955.

Vorosmarty, J. V., Meybeck, M., Fekete, B., Sharma, K., Green, P., and Syvitski, J. P. M. : Anthropogenic sediment retention : major global impact from registered river impoundments, Global Planet. Change, 39, 169-190, 2003.
Vilanova, I., Prieto, A. R., and Espinosa, M. : Palaeoenvironmental evolution and sea level fluctuations along the southeastern Pampa grasslands coast of Argentina during the Holocene, J. Quaternary Sci., 21, 227-242, 2006.

Zahar, Y., Ghorbel, A., and Albergel, J. : Impacts of large dams on downstream flow conditions of rivers : Aggradation and reduction of the Medjerda channel capacity downstream of the Sidi Salem dam (Tunisia), J. Hydrol., 351, 318-330, 2008. 